\title{
PREVALENCIA DE Neospora caninum EN PERROS PASTORES DE UNA EMPRESA GANADERA DE LA SIERRA SUR DEL PERÚ
}

\author{
Prevalence of Neospora caninum in Shepherd Dogs of a Livestock Farm in \\ THe Southern Highlands of Peru
}

\author{
Luis Vega O. ${ }^{1}$, Amanda Chávez V. ${ }^{1,2}$, Néstor Falcón P. ${ }^{3,4}$, Eva Casas A. ${ }^{1}$, \\ Nidia Puray Ch. ${ }^{1}$
}

\section{RESUMEN}

La neosporosis es una parasitosis que afecta a una gama de mamíferos domésticos y silvestres; destacando, por su importancia, la especie bovina, al producir problemas reproductivos como el aborto; y la especie canina, al actuar como hospedador definitivo y como fuente de diseminación de la enfermedad. El objetivo del presente trabajo fue determinar la prevalencia de Neospora caninum en perros pastores procedentes de cinco zonas de producción de la empresa Rural Alianza ubicada en el departamento de Puno. Se evaluaron 122 muestras de suero canino, recolectadas en los meses de febrero y marzo de 2004 y evaluadas mediante la prueba de Inmunofluorescencia Indirecta (IFI), en una dilución de 1:50. Se encontró una prevalencia de $14.8 \pm 6.3 \%$ (18/122). No se encontró diferencia estadística entre las variables procedencia (cinco sectores de la empresa), sexo y edad ( $<1,1$ a 7, $>7$ años), mediante la prueba de Chi Cuadrado. Este estudio demostró que los perros de la empresa Rural Alianza tuvieron contacto con Neospora caninum. Además, al comparar los valores hallados con estudios paralelos realizados en camélidos sudamericanos y bovinos de Puno, se evidenció una similitud en los valores de prevalencia entre los hospedadores intermediarios y definitivo.

Palabras clave: neosporosis, canino, IFI, prevalencia, transmisión

\section{Abstract}

Neosporosis is a parasitic disease that affects several domestic and wild mammals. Among them, it is important in the bovine species because affect reproduction causing abortions, and in canine species as it is the definitive host and a dissemination source for the disease. The aim of the present study was to determine the prevalence of Neospora caninum in shepherd dogs from five production areas of the Rural Alianza enterprise

\footnotetext{
${ }^{1}$ Laboratorio de Microbiología y Parasitología Veterinaria, ${ }^{3}$ Laboratorio de Medicina Veterinaria Preventiva, Facultad de Medicina Veterinaria, Universidad Nacional Mayor de San Marcos, Lima

${ }^{2}$ E-mail: achavezvg@gmail.com

${ }^{4}$ Dirección actual: Facultad de Veterinaria y Zootecnia, Universidad Peruana Cayetano Heredia, Lima
} 
located in the southern highlands, Puno. It was collected 122 samples of canine sera during February and March 2004, and they were analyzed using the Indirect Immunofluorescence assay (IFA), in a dilution of 1:50. A total prevalence of $14.8 \pm 6.3 \%$ (18/122) was found. Statistical association between rearing area (five areas of the farm), sex, and age ( $<1,1-7,>7$ years of age) through the chi square test was not found. This study showed that dogs from Rural Alianza showed a moderated prevalence for Neospora caninum. Besides, the prevalence reported is related to prevalence found for South American camelids and grazing cattle in Puno, which would indicate a close relationship between definitive and intermediate host, facilitating the horizontal transmission.

Key words: neosporosis, canine, IFA, prevalence, transmission

\section{INTRODUCCIÓN}

La neosporosis es una enfermedad parasitaria emergente causada por el protozoo Neospora caninum, perteneciente al phylum Apicomplexa. Actualmente se le reconoce como causante de trastornos neuromusculares en perros y abortos en bovinos (Dubey, 2003). Históricamente, en 1984 se notificó por primera vez a un protozoo como causante de miositis y encefalitis en seis cachorros en Noruega, los cuales resultaron serológicamente negativos a Toxoplasma gondii (Bjerkas et al., 1984). En 1988, casos similares fueron descritos en perros en Estados Unidos de América, a partir de los cuales se aisló al parásito que se denominó Neospora caninum y a la enfermedad como neosporosis (Dubey et al., 1988).

La importancia económica en el sector pecuario, se debe a que ocasiona pérdidas productivas y reproductivas, relacionadas con abortos, disminución en el número de crías y en la producción láctea, principalmente, en el ganado vacuno. En el Perú se ha confirmado las repercusiones económicas y sanitarias de esta enfermedad (Andresen, 1999), por lo que se han estado realizando estudios en especies nativas como los camélidos sudamericanos, reportándose prevalencias variadas. Sin embargo, hasta el momento, no se han demostrado las repercusiones económicas y sanitarias.
En el Perú existen estudios serológicos realizados en caninos. Así, se ha reportado una prevalencia de $32.7 \%$ en el valle de Lima (Del Campo et al., 2003), 28.9\% en la provincia de Chachapoyas (Horna et al., 2003), y $19.4 \%$ en el valle del Mantaro (Cornejo et al., 2004). Reportes en otros países indican una variada prevalencia; así se tiene $37.8 \%$ en Argentina (Basso et al., 2001), 11\% en Bélgica (Barber et al., 1997a), 8.3\% en Brasil (Canon-Franco et al., 2003) y 9\% en Australia, 22\% en Tanzania, y 20\% en Uruguay (Barber et al., 1997b).

En las zonas altoandinas se ha reportado infección de neosporosis en llamas (Moya et al., 2003) y en bovinos al pastoreo (Atoccsa et al., 2005). Sin embargo, no existen estudios serológicos en cánidos procedentes de estas zonas. El objetivo de este trabajo fue determinar la prevalencia de Neospora caninum en perros de cinco sectores de producción de la empresa Rural Alianza en el departamento de Puno, contribuyendo así a un mejor conocimiento de la epidemiología de la enfermedad en el país.

\section{Materiales y MéTodos}

\section{Ubicación del Estudio}

El presente trabajo se realizó en cinco sectores de las unidades de producción de la empresa Rural Alianza: Accopujio, Alianza, 
Antacalla, San Francisco y Conchatanca. Los cuatro primeros sectores se ubican en el distrito de Nuñoa, mientras que Conchatanca se ubica en el distrito de Macusani, provincias de Melgar y Carabaya, respectivamente, en el departamento de Puno. Esta empresa se ubica en la sierra sur del Perú, a una altitud que va de 3970 a $5200 \mathrm{msnm}$, y es una de las empresas líderes en el país en la crianza de camélidos sudamericanos; dedicándose además, a la producción de ovinos y bovinos.

\section{Tamaño Muestral}

Se estimó la población total de perros de la empresa Rural Alianza en base al número de pastores por cada sector, y considerando que cada pastor tiene 2-3 perros a su cargo. El tamaño muestral se determinó mediante la fórmula de población finita, usando una prevalencia referencial de 19.4\% (Cornejo et al., 2004) y un nivel de confianza de 95\% (Daniel, 1996), donde el número resultante fue de 112 perros. Además, la población a muestrear se estratificó por sector de producción, mediante la fórmula de estratificación de muestras (Daniel, 1996).

\section{Colección de Muestras}

Se colectaron muestras de sangre de 122 perros dedicados al pastoreo de camélidos sudamericanos, mayores de cinco meses, durante los meses de febrero y marzo de 2004. Las muestras de sangre se obtuvieron por punción de la vena cefálica y se recolectaron en tubos al vacío. El suero se extrajo el mismo día de la recolección y fue conservado a temperatura de congelación $\left(-20^{\circ} \mathrm{C}\right)$ hasta el día de su procesamiento. Se registró la edad, sexo y procedencia de los perros.

\section{Determinación de Anticuerpos}

Los anticuerpos contra Neospora caninum se determinaron mediante la prueba de inmunofluorescencia indirecta (IFI). Se usó una dilución de 1:50, utilizando como antígeno a taquizoítos formolizados (cepa Nc1) y conjugado comercial VMRD-USA. La positividad del suero fue considerada al observarse fluorescencia completa del taquizoíto, mientras que en la muestra negativa la fluorescencia es en forma parcial (apical), incompleta o nula (Paré et al., 1995).

\section{Análisis de Datos}

Se determinó la prevalencia de Neospora caninum utilizando la fórmula de Thrusfield (1990) y el intervalo de confianza mediante la fórmula de Armitage y Berry (1997). Se utilizó la prueba de Chi Cuadrado para determinar la asociación de las variables edad ( $<1,1$ a $7,>7$ años), sexo y procedencia (sector) con la presentación de la infección por $N$. caninum.

\section{Resultados}

La prevalencia de anticuerpos contra Neospora caninum en 122 muestras de sueros de caninos provenientes de cinco sectores de la empresa Rural Alianza fue de 14.8 $\pm 6.9 \%$ (18/122). En todos los sectores se observó la presencia de al menos un animal con anticuerpos contra N. caninum, siendo la frecuencia más alta en el sector de Conchatanca $(25 \%, 10 / 40)$ y la más baja en el sector de Antacalla (5.3\%, 1/19) (Cuadro 1).

Los animales menores de un año no presentaron anticuerpos contra $N$. caninum, siendo la frecuencia más alta en el grupo de 1 a 7 años con $17.5 \%$. Los perros machos presentaron una mayor frecuencia (15.7\%) con respecto a las hembras (10.0\%); sin embargo, ninguna de las variables en estudio (procedencia, sexo y edad) estuvieron estadísticamente asociadas con la frecuencia de infección (Cuadro 1).

\section{Discusión}

La neosporosis se ha convertido en una enfermedad emergente en diferentes partes 
Cuadro 1. Frecuencia de Neospora caninum en perros pastores de cinco sectores de producción de una empresa alpaquera en la zona de Puno, Perú, según procedencia, estrato etáreo y sexo (2004)

\begin{tabular}{|c|c|c|c|c|}
\hline Variable & & $\begin{array}{c}\text { Animales } \\
\text { muestreados }\end{array}$ & $\begin{array}{c}\text { Animales } \\
\text { seropositivos }\end{array}$ & $\begin{array}{c}\text { Frecuencia } \\
\text { (\%) }\end{array}$ \\
\hline \multicolumn{5}{|c|}{ Procedencia } \\
\hline & Alianza & 38 & 3 & 7.9 \\
\hline & Antacalla & 19 & 1 & 5.3 \\
\hline & Accopujio & 17 & 3 & 17.7 \\
\hline & Conchatanca & 40 & 10 & 25.0 \\
\hline & San Francisco & 8 & 1 & 12.5 \\
\hline \multicolumn{5}{|c|}{$\begin{array}{l}\text { Estrato etáreo } \\
\text { (años) }\end{array}$} \\
\hline & $<1$ & 16 & 0 & 0 \\
\hline & 1 a 7 & 97 & 17 & 17.5 \\
\hline & $>7$ & 9 & 1 & 11.1 \\
\hline \multicolumn{5}{|l|}{ Sexo } \\
\hline & Macho & 102 & 16 & 15.7 \\
\hline & Hembra & 20 & 2 & 10.0 \\
\hline Total & & 122 & 18 & $14.8 \pm 6.3^{1}$ \\
\hline
\end{tabular}

del mundo. Su transmisión se mantiene principalmente por la vía vertical (Wouda et al., 1999), y en menor escala por la vía horizontal en el caso de caninos (Trees et al., 2002), que actúan tanto como hospedador definitivo como intermediario. En la especie canina, la enfermedad se puede manifestar con la presentación de cuadros clínicos neuromusculares en perros jóvenes y, eventualmente, por abortos en las hembras gestantes (Dubey, 2003).

La prevalencia de anticuerpos contra $N$. caninum $(14.75 \%, 18 / 122)$ indica que los perros estuvieron expuestos al parásito en algún momento de su vida, ya sea en la etapa pre o post natal. Estudios similares realizados en caninos de otras zonas ganaderas del Perú muestran prevalencias variables; así tenemos en el Valle de Lima con 32.7\% (Del
Campo et al., 2003), en la provincia de Chachapoyas con 28.9\% (Horna et al., 2003) y en el Valle del Mantaro con 19.4\% (Cornejo et al., 2004). Al comparar los resultados del presente estudio con los resultados antes mencionados, se podría precisar que la prevalencia hallada fue moderada, y que existen factores como la convivencia estrecha de los perros con el ganado, número de perros por pastor, y condiciones climáticas, que estarían involucrados para que subsista la infección en la empresa Rural Alianza.

Los cánidos evaluados en el estudio, en contacto con camélidos sudamericanos (alpacas y llamas), presentaron prevalencias afines a las obtenidas en el ganado de la misma zona [16.7\% en llamas (Moya et al., 2003); $18.1 \%$ en bovinos al pastoreo (Atoccsa et al., 2005)], de allí que los factores de ries- 
go para la infección por este parasito serían similares. Asimismo, en estudios donde se dispone de resultados de seroprevalencia a $N$. caninum, tanto en ganado al pastoreo como en sus perros pastores, se evidencia esta correlación; así, en el valle de Lima, se encontró 32.7\% de frecuencia en caninos (Del Campo et al., 2003), mientras que en ganado vacuno se encontró $29.6 \%$ (Silva et al., 2002). Del mismo modo, en Chachapoyas, se encontró el $28.9 \%$ en caninos (Horna et al., 2003), mientras que en bovinos fue de 40.4\% (Quevedo et al., 2003). Otros estudios de comparación en caninos procedentes de zonas urbanas y rurales encontraron un mayor predominio en estos últimos, sugiriendo una exposicion postnatal al parásito (Basso et al., 2001).

La menor frecuencia de infección se presentó en el sector Antacalla (5.3\%, 1/19) y la mayor en Conchatanca (25\%, 10/40). A pesar de la aparente amplia diferencia entre las áreas evaluadas, no se encontró asociación estadística, debido posiblemente a no existir una dispersión homogénea del número de perros por sector de producción. Por otro lado, todos los sectores de producción realizan el mismo tipo de manejo de crianza extensiva, donde cada pastor tiene de 2 a 3 perros, que se desplazan libremente por las zonas de pastoreo, estando en contacto directo con el ganado; asimismo, las condiciones climáticas son relativamente similares entre ellas.

El grupo etáreo donde se encontró la mayor frecuencia de infección fue de 1 a 7 años (17.5\%), sin encontrar algún animal positivo en el grupo de perros menores de un año. No hubo diferencia estadística debido al sexo (machos: 15.7\%; hembras: 10.0\%). Estudios anteriores muestran similares resultados y señalan que el sexo no representa un factor de riesgo para la adquisición de la infección. (Basso et al., 2001; Del Campo et al., 2003; Horna et al., 2003; Cornejo et al., 2004).
La presencia de la neosporosis en la empresa Rural Alianza se debería, entre otros factores, a la presencia de perros pastores en las áreas de pastoreo del ganado, donde pueden ingerir fetos abortados, descargas uterinas y placentas de los hospedadores intermediarios, y de esa manera, facilitándose la transmisión del parásito (Dijkstra et al., 2002b); además, por la crianza mixta de camélidos con otras especies como ovinos y bovinos. Es justamente en estas especies, especialmente en bovinos, donde se ha confirmado la transmisión de la enfermedad al introducir un perro infectado con el parásito, ya que libera ooquistes al medio ambiente (Dijkstra et al., 2002a).

Otro factor a tomar en cuenta en la zona altoandina es la presencia de hospedadores silvestres, como es el zorro, que llega hasta las áreas de parición; ya que es posible la existencia de un ciclo de transmisión silvestre de $N$. caninum, al haberse confirmado al coyote como hospedador definitivo (Gondim et al., 2004). Así mismo, el zorro podría estar actuando como hospedador definitivo; sin embargo, esta teoría todavía no ha sido demostrada.

El presente trabajo aporta datos no existentes en el país, como la relación de la neosporosis canina con la crianza de camélidos sudamericanos en el altiplano, así como su aparente correlación con las prevalencias del ganado de la zona, lo que va permitir poder establecer medidas de control para evitar su transmisión.

\section{Conclusiones}

- La seroprevalencia de $N$. caninum en caninos de la empresa Rural Alianza fue moderada (14.8 $\pm 6.3 \%)$.

- La edad, sexo y procedencia (sector de producción) de los caninos infectados no fueron factores asociados con la posibilidad de adquirir $N$. caninum. 


\section{Literatura Citada}

1. Andresen H. 1999. Neosporosis en el Perú y el mundo. Rev Cienc Vet 15: 11-16.

2. Armitage P, Berry G. 1997. Statistical methods in medical research. $2^{\text {nd }} e d$. United Kingdom: Blackwell Scientific Publ. 597 p.

3. Atoccsa J, Chávez A, Casas E, Falcón N. 2005. Seroprevalencia de Neospora caninum en bovinos lecheros criados al pastoreo en la provincia de Melgar-Puno. Rev Inv Vet, Perú 16(1): 70-75.

4. Barber S, Van L, Ham I, Polis I, Trees J. 1997a. Seroprevalence of antibodies to Neospora caninum Belgian dogs. Small Anim Pract 38: 15-16.

5. Barber S, Gaser R, Ellis J, Reichel M, McMillan D, Trees J. 1997b. Prevalence of antibodies to Neospora caninum in different canid populations. J Parasitol 83: 1056-1058.

6. Basso W, Venturini L, Venturini MC, Moore P, Rambeau M, Unzaga JM, Campero C, et al. 2001. Prevalence of Neospora caninum infection in dogs from beef cattle farms, dairy farms, and from urban areas of Argentina. J Parasitol 87: 906-907.

7. Bjerkas I, Mohn S, Presthus J. 1984. Unidentified cystforming sporozoon causing encephalomyelitis and myositis in dogs. Z Parasitenkd 70: 271-274.

8. Canon-Franco W, Bergamaschi D, Labruna M, Camargo L, Souza S, Silva J, Pinter A, et al. 2003. Prevalence of antibodies to Neospora caninum in dogs from Amazon, Brazil. Vet Parasitol 115: 71-74.

9. Cornejo N, Chávez A, Casas E, Arana C. 2004. Seroprevalencia de Neospora caninum en perros de establos lecheros de la cuenca izquierda del Valle del Mantaro. Rev Inv Vet, Perú 15(1): 70-75.

10. Daniel W. 1996. Bioestadística: base para el análisis de la ciencia de la salud. $5^{\text {ta }}$ ed. México: Ed. Limusa. 878 p.
11. Del Campo J, Chávez A, Delgado A, Falcón N, Ornelas A, Casas E, Serrano E. 2003. Frecuencia de Neospora caninum en perros de establos lecheros del valle de Lima. Rev Inv Vet, Perú 14(2): 145-149.

12. Dijkstra T, Barkema H, Hesselink J, Wouda W. 2002a. Point source exposure of cattle to Neospora caninum consistent with periods of common housing and feeding and related to the introduction of a dog. Vet Parasitol 105: 89-98.

13. Dijkstra T, Barkema H, Eysker M, Hesselink J, Wouda W. 2002b. Natural transmission routes of Neospora caninum between farm dogs and cattle. Vet Parasitol 105: 99-104.

14. Dubey J. 2003. Review of Neospora caninum and neosporosis in animals. Korean J Parasitol 41: 1-16.

15. Dubey J, Carpenter J, Speer C, Topper M, Uggla A. 1988. Newly recognized fatal protozoan disease of dogs. J Am Vet Med Assoc 192: 12691285.

16. Gondim L, McAllister M, Pitt W, Zemlicka D. 2004. Coyotes (Canis latrans) are definitive hosts of Neospora caninum. Int J Parasitol 34: 159-161.

17. Horna M, Chávez A, Rivera $\mathrm{H}, \mathrm{Ca}$ sas E, Serrano E. 2003. Seroprevalencia de Neospora caninum en caninos en dos distritos de la provincia de Chachapoyas. Rev Inv Vet, Perú 14: 150-154.

18. Moya R, Chávez A, Casas E, Serrano E, Falcón N, Pezo D. 2003. Seroprevalencia de Neospora caninum en llamas de la provincia de Melgar, Puno. Rev Inv Vet, Perú 14: 155-160.

19. Paré J, Hietala S, Thumond M. 1995. Interpretation of and indirect fluorescence antibody test for diagnosis of Neospora spp. infection in cattle. $\mathrm{J}$ Vet Diagn Invest 7: 273-275.

20. Quevedo J, Chávez A, Rivera H, Casas E, Serrano E. 2003. Neosporosis en bovinos lecheros en dos distritos de 
la provincia de Chachapoyas. Rev Inv Vet, Perú 14: 33-37.

21. Silva P, Chávez A, Rivera H, Casas E. 2002. Seroprevalencia de Neospora caninum en bovinos lecheros del Valle de Lima. Rev Inv Vet, Perú 13: 51-55.

22. Thrusfield M. 1990. Epidemiología veterinaria. Zaragoza, España: Ed. Acribia. 339p.

23. Trees AJ, McAllister MM, Guy CS, Smith R, Williams DJL. 2002. Oral infection of pregnant cows with Neospora caninum oocysts. Res Vet Sci 72(Supl 1): 34 (Abstr).

24. Wouda W, Bartels CJM, Moen AR. 1999. Characteristics of Neospora caninum associated to abortion epidemics in dairy herds in The Netherlands (1995 to 1997). Theriogenology 52: 233-245. 